\title{
Molecular analysis of T cell receptor $\beta$ variability in a patient with orofacial granulomatosis
}

\author{
S H Lim, P Stephens, Q-X Cao, S Coleman, D W Thomas
}

\begin{abstract}
Background-Orofacial granulomatosis (OFG) is a rare chronic inflammatory disorder of unknown causation and is characterised histologically by noncaseating granulomas and aggregates of small lymphocytes. The molecular nature of these $\mathbf{T}$ cells is, however, unclear.

Aims-To determine the $\mathrm{T}$ cell receptor (TCR) V $\beta$ gene usage of the $T$ cell infiltrate associated with the primary lesions in a patient with OFG.

Methods-A molecular method involving reverse transcriptase (RT)-polymerase chain reaction (PCR), DNA cloning, single strand conformation polymorphism (SSCP), length analysis, and nucleotide sequencing was used.

Results-Compared with peripheral blood lymphocytes from the same patient, notably restricted TCRV $\beta$ gene usage was observed in the $T$ cell infiltrate. Only three of the 24 major TCRV $\beta$ gene families were represented in the repertoire. There was preferential usage of the VB6 gene. In addition, more than $20 \%$ of the VB6 TCR transcripts exhibited an identical unique V-D-J junctional sequence, suggesting a local antigen driven VB6 $T$ cell clonal expansion in vivo, a phenomenon not observed in normal oral mucosa.

Conclusions-The TCRV $\beta$ repertoire of T cells associated with OFG is restricted. It is also associated with a local $T$ cell clonal expansion. The results, therefore, provide a new perspective on the immunopathology of OFG.

(Gut 1997; 40: 683-686)
\end{abstract}

Department of Haematology, University of Wales College of Medicine $S$ H Lim

Q-X Cao

$S$ Coleman

Department of Oral Medicine, Surgery and Pathology, Cardiff Dental School

P Stephens

D W Thomas

Correspondence to:

Dr Seah H Lim

Department of Haematology, University of Wales College of Medicine, Heath Park, Cardiff CF4 4XN.

Accepted for publication 28 November 1996
Keywords: T cell receptor, orofacial granulomatosis, $\mathrm{V} \beta$ gene usage.

Orofacial granulomatosis (OFG) is a chronic inflammatory disorder typically affecting young adults and causing swelling of the lips, cheeks and tongue. Various observations suggest that OFG may be immunologically mediated. These observations include associations with allergy to food additives ${ }^{1}$ and cobalt, ${ }^{2}$ increased circulating immune complexes $^{3}$ and antibody titres to the mycobacterial stress protein, mSP65, ${ }^{4}$ and an abnormal CD4:CD8 ratio. ${ }^{5}$ Positive Kveim reactions have also been observed in some patients. ${ }^{6}$ Furthermore, immunosuppressive agents such as corticosteroids ${ }^{7}$ produce remission of the disease.

Histologically, OFG resembles Crohn's disease and is characterised by non-caseating epitheloid granulomas with or without multinucleated giant cells. There is frequently oedema of the superficial corium with lymphangiectasia, and both diffuse and focal aggregates of small lymphocytes. Analysis of the $T$ cell receptor (TCR) $\beta$ variability $(V)$ and clonality of the lesional lymphocytes associated with the granuloma may therefore provide clues to the nature of the immune reaction.

\section{Case report}

A 12 year old boy presented with a six month history of diffusely swollen lips, associated with a painful, bilateral angular cheilitis. Examination revealed the presence of generalised swelling of his upper and lower lips, a notable desquamative gingivitis and mucosal tagging in the right buccal mucosa. A $6 \mathrm{~mm} \times 5 \mathrm{~mm}$ incisional biopsy specimen of his right buccal mucosa revealed the presence of epitheloid cell granulomas with many multinucleated giant cells. No acid-alcohol fast bacilli were identified on Ziehl-Neelsen staining. A diagnosis of OFG was made. The patient was treated with a miconazole gel for his angular cheilitis and was placed on a benzoate and cinnamon free diet. His gingivitis and lip swelling resolved within three months of presentation.

\section{Methods}

RT-PCR AND SOUTHERN ANALYSIS OF TCRV $\beta$ REPERTOIRE

The method used was as described previously. ${ }^{8}$ Briefly, total RNA was extracted from the oral mucosa biopsy specimen after cell lysis with a guanidinium isothiocyanate buffer by phenol and chloroform. ${ }^{9}$ The integrity of the isolated RNA was confirmed on a formaldehyde denaturing agarose gel. First strand cDNA was synthesised from total RNA using random hexamer primers and reverse transcriptase (Perkin Elmer, Roche Molecular Systems Inc., NJ, USA). For analysis and PCR amplification of TCRV $\beta$ gene segments, 25 oligonucleotides specific for the 24 known major $V \beta$ families 
were used as the $5^{\prime}$ primers and a $C \beta$ specific oligonucleotide as the $3^{\prime}$ primer (Clontech Laboratories Inc., CA, USA) in a $50 \mu l$ PCR reaction mixture. The hot start PCR method was used at $94^{\circ} \mathrm{C}$ to reduce non-specific priming. PCR conditions were as follows: $94^{\circ} \mathrm{C}$ for one minute, $55^{\circ} \mathrm{C}$ for one minute and $72^{\circ} \mathrm{C}$ for one minute for 35 cycles. The positive control amplified a segment of $\mathrm{C} \alpha$ and the negative control was comprised of the PCR reaction mixture lacking cDNA. Amplification products $(25 \mu \mathrm{l})$ were electrophoresed on a $2 \%$ agarose gel (200 volts for two hours) and visualised by ultraviolet illumination for DNA fragments of the predicted sizes of between 190 and $350 \mathrm{bp}$. The specificity of the amplified products was confirmed by Southern blotting onto a Biodyne-B transfer membrane (Pall Europe Limited, UK) and hybridisation overnight at $55^{\circ} \mathrm{C}$ with a $\mathrm{C} \beta$ internal probe (Clontech Laboratories) end-labelled with $\left[\gamma-{ }^{32} P\right]$ ATP. Negative control for $C \beta$ probe hybridisation consisted of the $\mathrm{C} \alpha$ amplification product. The membrane was washed with $2 \times$ SSC and $0.05 \%$ SDS at room temperature for 20 minutes and autoradiographed with intensifier for four hours at $-70^{\circ} \mathrm{C}$ before being developed and analysed.

\section{CLONING OF THE PCR PRODUCT}

The PCR product was cloned using a TA cloning kit (Invitrogen Corporation, USA). Briefly, the PCR product was ligated into the pCR ${ }^{\mathrm{TM}} \mathrm{II}$ TA cloning site which is flanked by two EcoR1 restriction sites. Competent Escherichia coli (INV $\alpha \mathrm{F}^{\prime}$ cells) were transformed by heat shock and plated for ampicillin and blue-white colour selection on agar plates containing 5-bromo-6-chloro-3-indolyl$\beta$-D-galactopyranoside ( $x-\mathrm{Gal}$ ).

\section{SINGLE STRAND CONFORMATION}

POLYMORPHISM (SSCP) ANALYSIS

Equal volumes of $75 \%$ glycerol and DNA were mixed, heated to $95^{\circ} \mathrm{C}$ for five minutes and electrophoresed on a $6 \%$ polyacrylamide gel (200 volts for two hours). The DNA was visualised by staining with silver nitrate.

\section{DNA LENGTH ANALYSIS}

Formamide loading buffer $(4 \mu \mathrm{l})$ was added to $10 \mu \mathrm{l}$ of each plasmid digested with EcoR1 restriction enzyme. The resultant mixture was heated to $75^{\circ} \mathrm{C}$ for five minutes and electrophoresed on a $6 \%$ polyacrylamide/urea gel (200 volts for two hours). The DNA was visualised by staining with silver nitrate.

\section{SILVER NITRATE STAINING}

The gel was first washed in water for five minutes. This was followed by sequential incubation in $0.1 \%$ nitric acid for five minutes, water for 20 seconds, silver nitrate solution $(100 \mathrm{mg}$ of silver nitrate in $100 \mathrm{ml}$ water and $100 \mu \mathrm{l} 40 \%$ formaldehyde solution) for 20 minutes, and water for five minutes. Finally, the gel was developed in sodium carbonate solution $(7.5 \mathrm{~g}$ sodium carbonate in $250 \mu \mathrm{l}$ water and $125 \mu \mathrm{l} 40 \%$ formaldehyde solution) until the DNA was visualised.

NUCLEOTIDE SEQUENCING

Plasmid DNA was extracted from positive colonies. Single strand templates were prepared by alkaline denaturation of the purified plasmid. Samples were sequenced using the dideoxy-chain termination procedure incorporating Sequenase 2.0 (United States Biochemicals, Cleveland, OH, USA) and $\left[\alpha^{-{ }^{35}} \mathrm{~S}\right]$ dATP.

\section{Results and Discussion}

In this study, our aim was to investigate the TCRV $\beta$ gene usage in the lesional lymphocytes in a case of OFG to determine the nature of the immune response and small lymphocytes associated with OFG. We reasoned that if OFG is immunologically mediated, a specific immune response may be observed and reflected in the TCRV $\beta$ gene usage by the lesional lymphocytes. TCRV $\beta$ gene expression can be probed either at the protein level by use of monoclonal antibodies or at the mRNA level using various methods based on oligonucleotide driven PCR amplification. We chose to use a molecular method instead of monoclonal antibodies to permit a more sensitive and comprehensive analysis of the $T$ cell repertoire as well characterised monoclonal antibodies are still not available for the full complement of 24 major TCRV $\beta$ families. Molecular analysis also permits the identification of a $T$ cell population at a clonal level. We analysed the TCRV $\beta$ repertoire of the lesional lymphocytes in situ without in vitro separation from the biopsy tissue and culture of these lymphocytes in order to avoid any effect the separation and culture may have on any particular clones or families of $T$ cells.

Using a panel of family specific primers in a sensitive and specific method of RT-PCR, we have compared TCRV $\beta$ gene usage by the lesional lymphocytes with the peripheral blood lymphocytes (PBL) from the same patient. We also compared the lesional lymphocytes with those obtained from normal oral mucosa from three healthy individuals. Unlike the TCRV $\beta$ repertoire of normal oral mucosa, ${ }^{10}$ which showed usage of a full complement of the 24 major TCRV $\beta$ families, we observed in this study that the TCRV $\beta$ gene usage by the lesional lymphocytes in OFG was notably restricted. This was confirmed by two independent RT-PCR assays. Only three of the 24 major gene families were used $(V \beta 3, V \beta 4$ and $V \beta 6$ ). There was also preferential usage of the VB6 family of genes in the lesional lymphocytes (Fig 1A). This is in contrast to PBL in which a near full complement of the $\mathrm{V} \beta$ gene families was used (Fig 1B), suggesting that the local T lymphocyte recruitment in OFG was not random.

Restricted TCRV $\beta$ gene usage can arise as a result of local $T$ cell clonal expansion or 
A

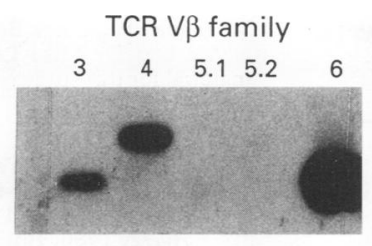

B

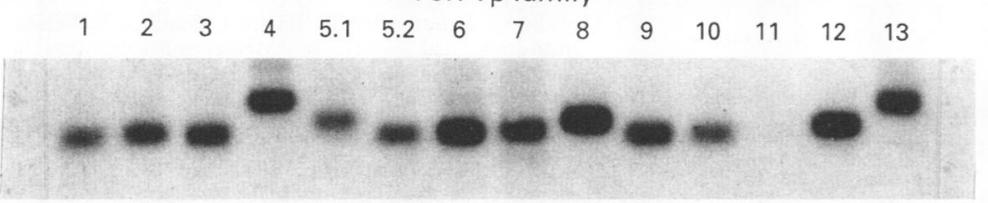

$\begin{array}{lllllllllll}14 & 15 & 16 & 17 & 18 & 19 & 20 & 21 & 22 & 23 & 24\end{array}$

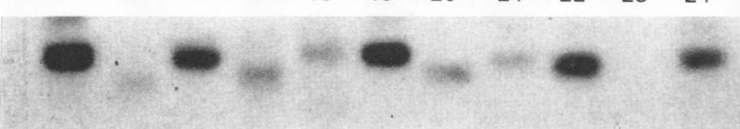

Figure 1: Southern blotting and hybridisation of the PCR products using a $\left[\gamma^{32} \mathrm{P}\right]$ ATP labelled internal $C \beta$ probe. (A) Only VB3, 4 and 6 were detected in the lesional lymphocytes, with VB6 being most prominently expressed. (B) In contrast, nearly the full complement of the $24 T C R V \beta$ major families were represented in the $P B L$

\section{V $\beta 6$ colony}

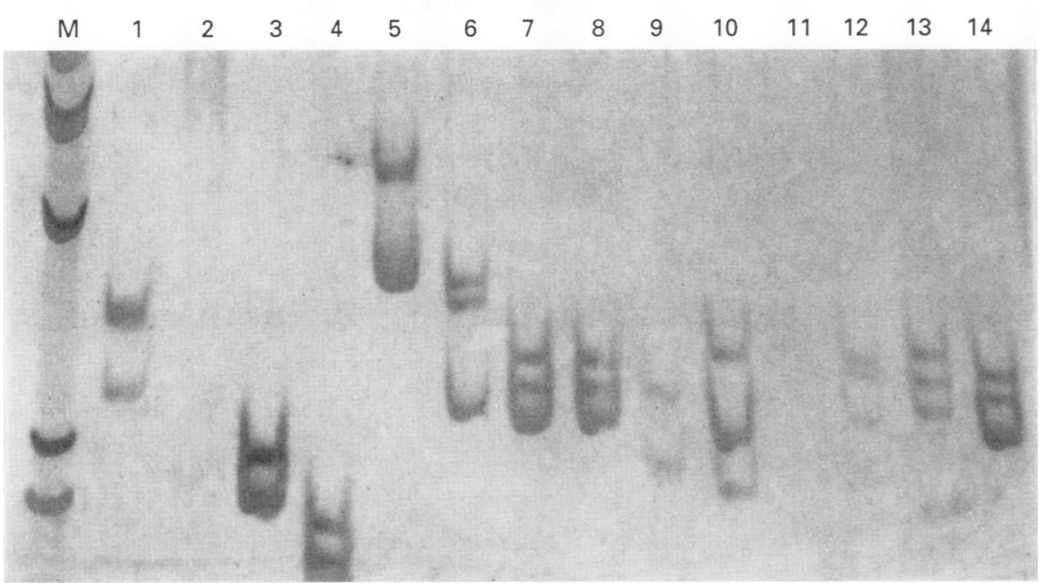

Figure 2: SSCP analysis of the 14 recombinant plasmids digested with EcoR1, indicating that three of 14 were identical. ( $M=1$ kilobase ladder).

\section{V $\beta 6$ colony}

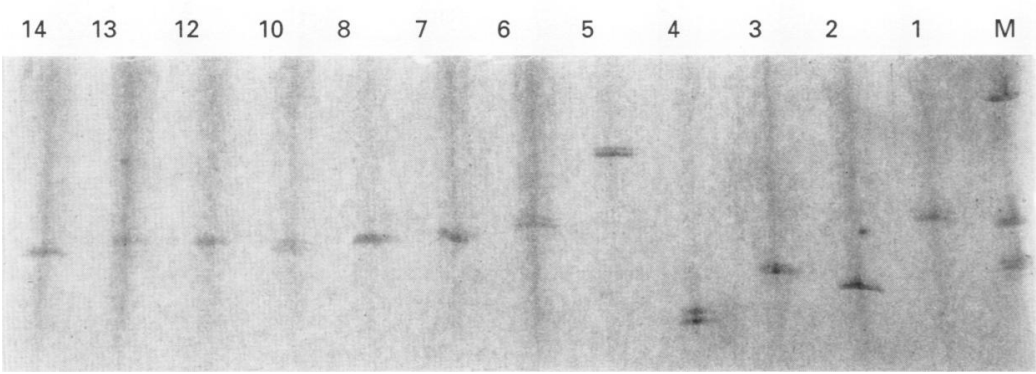

Figure 3: DNA length analysis of 12 recombinant plasmids digested with EcoR1, indicating that only four of 14 were identical. ( $M=$ single strand 1 kilobase ladder).

superantigen mediated TCRV $\beta$ family specific $T$ cell recruitment, or both. In order to determine the clonality of the V $\beta 6 \mathrm{~T}$ cells present in association with OFG, the lymphocytes were characterised further by cloning of the PCR products from the lesional lymphocytes and TCRV $\beta 6$ PBL from the same patient. Fourteen white colonies were randomly picked from the successful trans- formants from lesional lymphocytes and 10 from $\mathrm{PBL}$, and they were then expanded for individual plasmid preparation. Recombinant plasmids were first confirmed in all 24 colonies by restriction digestion with EcoR1, which produced DNA fragments of between 200 and 250 bp (data not shown). Recombinant plasmids digested with EcoRl were then used for SSCP and DNA length analysis. Figure 2 shows that three $(21 \%)$ of 14 of the TCRV $\beta 6$ clones from lesional lymphocytes exhibited an identical SSCP pattern (colonies 7, 8 and 13). DNA length analysis carried out on 12 colonies (colonies 9 and 11, which did not show an identical SSCP pattern, were not analysed because there was insufficient DNA) indicated that colonies $7,8,12$ and 13 were identical in length (Fig 3). Interestingly, three of these four DNA fragments also shared an identical SSCP pattern, suggesting that they might be identical in their nucleotide sequence.

The four TCRV 36 cDNAs showing identical lengths were sequenced. Three colonies (7, 8 and 13) produced an identical unique nucleotide sequence (Table). Colony 12 gave an entirely different sequence (data not shown). The Table shows deduced in-frame amino acid sequence of the V-D-J junction of the three identical TCRV $\beta 6$ PCR products. $\mathrm{J} \beta 2.1$ and $\mathrm{C} \beta 2$ genes were being used in the V-D-J-C rearrangement. Together, these results identified a recurrent TCRV $\beta$ sequence in at least $20 \%$ of the V $\beta 6$ lesional lymphocytes. The finding of a recurrent sequence indicates an increase in the copy number of the mRNA encoding the sequence in the initially prepared total RNA and suggests an antigen driven $\mathrm{V} \beta 6 \mathrm{~T}$ cell clonal expansion taking place in vivo. Such $T$ cell clonal expansion only occurred locally as none of the 10 colonies from the V $\beta 6$ PBL exhibited an identical SSCP pattern or DNA length when they were compared with one of the identical colonies derived from the lesional lymphocytes (data not shown). To exclude the possibility that recurrent V $\beta 6$ transcript is normally observed in oral mucosa, we analysed between 17 and 20 recombinant colonies from each of the TCRVB6 PCR products from the three samples of normal oral mucosa and none showed any transcript with identical DNA length and SSCP pattern (data not shown), suggesting the absence of a V $\beta 6$ recurrent transcript in normal oral mucosa.

In conclusion, we have demonstrated in this study that TCRV $\beta$ gene usage by the lesional $T$ lymphocytes is notably restricted in a patient with OFG. In addition, a recurrent transcript in the V $\beta 6$ lesional lymphocytes was identified, suggesting a clonal $\mathrm{T}$ cell expansion in the vicinity of the lesion. The local, restricted TCRV $\beta$ gene usage and clonal $T$ cell expansion was only observed in the lesional lymphocytes, and is likely to have been the result of OFG as restricted TCRV $\beta$ gene usage or recurrent transcript was not observed in normal oral mucosal lymphocytes. The biological role of these $T$ cells remains to be determined. However, it is likely that the restricted $T$ cell repertoire and clonal $T$ cells 
Nucleotide (lower case) and the deduced in-frame V-D-F junctional amino acid (upper case) sequence of the three identical colonies. Primer sequences are in bold, italics and underlined

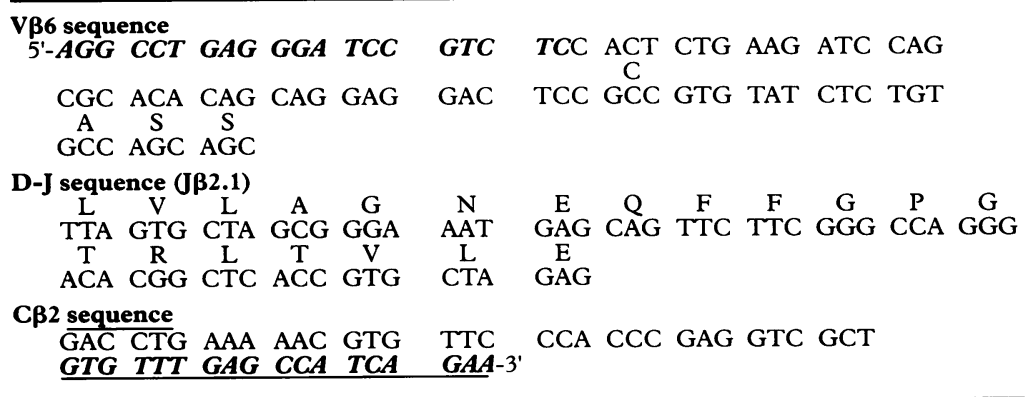

are involved in a delayed hypersensitivity reaction in response to an antigen. Local cytokine release may be responsible for the characteristic histological features of OFG. Although the exact antigen eliciting a $\mathrm{T}$ cell immune response remains unknown, our preliminary data provide a new perspective for the immunopathology of OFG. Further similar work is needed to define the immunology in this rare condition.
We thank Dr Derrick Bowen for his most constructive technical advice and Dr Mike Lewis for providing us with the clinical material for this study.

1 Sweatman MC, Tasker R, Warner JO, Ferguson MM, Mitchell $\mathrm{D}$. Oro-facial granulomatosis. Response to elemental diet and proveca

2 Pryce D, King CM. Oro-facial granulomatosis associated with delayed hypersensitivity to cobalt. Clin Exp Dermato 1990; 15: 384-6.

3 Morales C, Penarrocha M, Bagan J, Burches E, Pelaez A Immunological study of Melkersson-Rosenthal syndrome. Lack of response to food additive challenge. Clin Exp Allergy 1995; 25: 260-4.

4 Ivanyi $L$ Kirby A, Zakrzewska JM. Antibodies to mycobacterial stress protein in patients with orofacial granulomatosis. F Oral Pathol Med 1993; 22: 320-2.

5 Henry CH. Orofacial granulomatosis: report of a case with decreased CD4/CD8 ratio. $\mathcal{F}$ Oral Maxillofacial Surg 1994; 52: 317-22

6 Schehade SA, Foulds IS. Granulomatous cheilitis and a positive Kveim test. Br f Dermatol 1986; 115: 619-22.

7 Wiesenfeld D, Ferguson MM, Mitchell DN, MacDonald DG, Scully C, et al. Oro-facial granulomatosis - a clinical and pathological analysis. $Q \mathcal{F} \mathrm{Med}$ 1985; 54: 101-13.

8 Coleman S, Perera, Fisher J, Hoy T, Burnett AK, LimSH Abnormal TCR V $\beta$ repertoire in patients with chronic myeloid leukaemia. Br $\mathcal{F}$ Haematol 1995; 90: 358-63.

9 Chomecynski P, Sacchi N. Single-step method of RNA isolation by acid guanidium thiocyanate-phenol-chloroisolation by acid guanidium thiocyanate-phen

$10 \mathrm{Lim} \mathrm{H}$, Thomas DW, Coleman S, Stephens P. T-cell receptor V $\beta$ repertoire of tumour infiltrating lymphocytes in oral squamous cell carcinoma. Cancer Immunol Immunother 1996; 42: 69-70. 